\title{
Characterisation of optimal dual measures via distortion
}

\section{Michael Monoyios}

Mathematical Institute, University of Oxford

e-mail: monoyios@maths.ox.ac.uk

Received: 14 April 2005/ Accepted: 22 May 2006 - (C) Springer-Verlag 2006

\begin{abstract}
We derive representations for optimal martingale measures in a two-factor Markovian model, by seeking ramifications of a distortion power solution (Zariphopoulou (2001)) of the primal utility maximisation problem, for the dual problem. This provides an alternative to existing methods in the literature for characterising optimal measures, and gives new results in the form of a novel representation for the dual stochastic control problem, and in the form of Esscher transform relations between the optimal measure and the minimal measure.
\end{abstract}

Journal of Economic Literature Classification: C61, D52, G11, G13

Mathematics Subject Classification (2000): 58J37, 60H30, 93E20

\section{Introduction}

This paper analyses optimal measures in a two-factor Markovian market containing a stock $S$ and a stochastic volatility $Y$, driven by two correlated Brownian motions $W, \widetilde{W}$, respectively, with fixed correlation $\rho \in[-1,1]$. The parameters of the stochastic differential equation for $S$ are progressively measurable with respect to the filtration $\widetilde{\mathbb{F}}:=\left(\widetilde{\mathcal{F}}_{t}\right)_{0 \leq t \leq T}$ generated by $\widetilde{W}$. We exploit an explicit solution, known as a distortion power solution (Zariphopoulou (2001), Tehranchi (2004)), for a primal utility maximisation problem, and seek ramifications for the dual to the primal problem.

In the distortion power solution, a value function $u(x)=E U\left(X_{T}^{*}\right)$, in which $U$ is the utility function, $X_{T}^{*}$ is optimal terminal wealth and $x$ is initial wealth, is obtained in the form $u(x)=U(x)\left[E^{\widetilde{P}^{M}} \zeta^{1 / \delta}\right]^{\delta}$. Here, $\zeta$ is an $\widetilde{\mathcal{F}}_{T}$-measurable random variable, $\delta$ is known as the distortion power and 
depends on the correlation $\rho$ (and, with power utility, on the risk aversion parameter), and $\widetilde{P}^{M}$ is a measure related to the minimal martingale measure $Q^{M}$ (and which, with exponential utility, is the projection of $Q^{M}$ onto $\widetilde{\mathcal{F}}_{T}$ ). Our results stem from translating this representation into a representation for the solution of the dual problem. The random variable $\zeta$ is of the form $H\left(0, Y_{0}\right)$ for some function $H$ which satisfies, by the Feynman-Kac theorem, a linear parabolic PDE.

The motivation for this work is, first, to understand the relations (if any) between the distortion method and the dual approach, and between the optimal martingale measure $Q^{*}$ of the dual problem and the measure $\widetilde{P}^{M}$ in the distortion solution. Second, we derive a number of representations for $Q^{*}$ using the distortion solution, for both power and exponential utility, and our methods are an alternative to some existing techniques in the literature, notably the representation equations of Hobson (2004), Rheinländer (2005), and the PDE representation of Benth and Karlsen (2005).

The main results are as follows. First, we derive a general representation for $Q^{*}$ in terms of $\zeta=H\left(0, Y_{0}\right)$ (Lemma 1) by translating the distortion solution into a formula for the dual value function. This is the initial relation between $Q^{*}$ and $\widetilde{P}^{M}$, and is used frequently in what follows.

Suppose the dual optimiser has density with respect to the physical measure $P$ given by $d Q^{*} / d P=\mathcal{E}\left(-\lambda \cdot W-\xi \cdot W^{\perp}\right)$, where $\lambda$ is the Sharpe ratio of the stock, $W^{\perp}$ is a Brownian motion independent of $W$, and $\xi$ is an optimal integrand characterising $Q^{*}$. We derive an expression (Theorem 1) for $\xi_{t}$ in terms of $H\left(t, Y_{t}\right)$. This generalises a result of Benth and Karlsen (2005) for the minimal entropy measure to encompass the dual measure associated with a power utility function.

We then show that the above formula for $\xi$ gives a solution to the representation of Hobson (2004) for $Q^{*}$ (Proposition 3), and we use this to verify optimality of $\xi$ for the dual problem. By duality, this implies optimality of the trading strategy $\pi^{*}$ in the primal problem, that is associated with the distortion solution. We then derive the relationship between $\pi^{*}$ and $\xi$ (Proposition 4).

Viewing $\xi$ as the optimal control of a dual stochastic control problem, we then give a novel representation for the solution to this control problem (Theorem 2). To the best of our knowledge this result is new. The dual control problem is to optimise a functional $E^{P^{\psi}} C^{\psi}$, where $\psi$ represents the control, and where the measure $P^{\psi}$ and integral $C^{\psi}$ both depend on $\psi$. The distortion solution implies that the solution of the dual control problem is of the form $\Phi\left(E^{P^{\psi}} C^{\psi}\right)=E^{P^{0}} \Phi\left(C^{0}\right)$, where $\Phi$ is a given function and $P^{0}$ (when projected onto $\widetilde{\mathcal{F}}_{T}$ ) is the measure $\widetilde{P}^{M}$ in the distortion solution.

Finally, our analysis culminates in an extremely succinct relationship between $Q^{*}$ and $\widetilde{P}^{M}$ (Theorem 3), in the form of an Esscher transform relation 
between a measure $P^{k \xi}$ and $\widetilde{P}^{M}$, where $k$ is a constant depending on the correlation (and $P^{\psi}$ is the measure appearing in the dual control problem). For exponential utility this result reduces to the beautiful result that the projections of the minimal entropy measure $Q^{E}$ and the minimal martingale measure $Q^{M}$ onto $\widetilde{\mathcal{F}}_{T}$ are related by an Esscher transform (Corollary 3$)$. This relationship between $Q^{E}, Q^{M}$ in models of this type appears to be new. It may be viewed as quantifying the economic premium associated with exponential valuation, relative to that associated with quadratic or logarithmic criteria.

The rest of this paper is as follows. The next section describes the market model, Section 3 gives the distortion solution, Section 4 derives our main results (representations for $Q^{*}$ ), and Section 5 briefly applies the same program to a problem with random endowment, under exponential utility.

\section{The market model}

Let $(\Omega, \mathcal{F}, P)$ be a probability space supporting two correlated Brownian motions $W:=\left(W_{t}\right)_{0 \leq t \leq T}$ and $\widetilde{W}:=\left(\widetilde{W}_{t}\right)_{0 \leq t \leq T}$ with fixed correlation $\rho \in$ $[-1,1]$. Let $\mathbb{F}:=\left(\mathcal{F}_{t}\right)_{0 \leq t \leq T}$ be the completion of the filtration generated by the pair $(W, \widetilde{W})$, and denote by $\widetilde{\mathbb{F}}:=\left(\widetilde{\mathcal{F}}_{t}\right)_{0 \leq t \leq T}$ the completion of the filtration generated by $\widetilde{W}$.

The model comprises a traded asset $S:=\left(S_{t}\right)_{0 \leq t \leq T}$ and a stochastic volatility $Y:=\left(Y_{t}\right)_{0 \leq t \leq T}$, described by the stochastic differential equations

$$
\begin{aligned}
& d S_{t}=Y_{t} S_{t}\left(\lambda\left(Y_{t}\right) d t+d W_{t}\right), \\
& d Y_{t}=a\left(Y_{t}\right) d t+b\left(Y_{t}\right) d \widetilde{W}_{t},
\end{aligned}
$$

under the physical measure $P$, and subject to initial conditions. We write $\widetilde{W}_{t}=\rho W_{t}+\bar{\rho} W_{t}^{\perp}$, with $\bar{\rho}=\sqrt{1-\rho^{2}}$, and in which $\left(W, W^{\perp}\right):=$ $\left(W_{t} W_{t}^{\perp}\right)_{0 \leq t \leq T}$ is a two-dimensional Brownian. Note that the parameter functions $\lambda, a, b$ are $\widetilde{\mathbb{F}}$-adapted.

We assume that $\lambda, a, b$ are such that unique strong solutions to the stochastic differential equations (1-2) exist. A Lipschitz condition is sufficient, but we do not impose this on the parameters at this stage, as we do not wish to exclude some well-known stochastic volatility models from the outset.

The interest rate is zero, or equivalently $S$ represents a discounted price. This entails no loss of generality if interest rates are deterministic. Stochastic interest rates are a non-trivial extension, as the choice of numéraire can yield differing optimal investment problems.

In general, the information available to an agent at $t \in[0, T]$ is $\mathcal{F}_{t}^{S}=$ $\sigma\left(\left\{S_{s} ; s \leq t\right\}\right)$. However, $Y_{t}^{2}$ is clearly measurable with respect to $\mathcal{F}_{t}^{S}$. 
If, further, $Y_{t}$ is measurable with respect to $\mathcal{F}_{t}^{S}$, for example, if $Y_{t}$ is necessarily non-negative, then $Y_{t}$ is $\mathcal{F}_{t}^{S}$-measurable, and so $W_{t}, \widetilde{W}_{t}, W_{t}^{\perp}$ are $\mathcal{F}_{t}^{S}$-measurable, and we have $\mathcal{F}_{t}^{S}=\mathcal{F}_{t}$. Henceforth we assume this is the case, though it rules out some cases in the literature. Rheinländer (2005) discusses this issue in some depth with particular reference to a version of the Stein-Stein (1991) model.

The mean-variance trade-off is the increasing process $K:=\left(K_{t}\right)_{0 \leq t \leq T}$ given by

$$
K_{t}:=\int_{0}^{t} \lambda^{2}\left(Y_{s}\right) d s, 0 \leq t \leq T .
$$

We assume throughout that $K_{t}<\infty, 0 \leq t \leq T, P$-almost surely. In what follows we sometimes write $\lambda_{t} \equiv \lambda\left(Y_{t}\right), 0 \leq t \leq T$, and $(\lambda \cdot W)_{t}$ for the integral $\int_{0}^{t} \lambda_{s} d W_{s}$.

The class $\mathcal{M}$ of local martingale measures is the set of measures $Q$ with density processes

$$
\left.\frac{d Q}{d P}\right|_{\mathcal{F}_{t}} \equiv Z_{t}:=\mathcal{E}\left(-\lambda \cdot W-\psi \cdot W^{\perp}\right)_{t}, 0 \leq t \leq T,
$$

where $\mathcal{E}$ is the Doléans exponential, and $\psi$ is an $\mathbb{F}$-adapted process satisfying $\int_{0}^{T} \psi_{t}^{2} d t<\infty P$-almost surely. If $\lambda, \psi$ are such that $E Z_{T}=1$, then the local $(P, \mathbb{F})$-martingale $Z:=\left(Z_{t}\right)_{0 \leq t \leq T}$ is a true martingale and $Q \in \mathcal{M}$ are probability measures equivalent to $P$ on $\mathcal{F}_{T}$.

The $P$-dynamics of $Z$ are

$$
d Z_{t}=-\lambda\left(Y_{t}\right) Z_{t} d W_{t}-\psi_{t} Z_{t} d W_{t}^{\perp} .
$$

Under $Q \in \mathcal{M}, d S_{t}=Y_{t} S_{t} d W_{t}^{Q}$, and $Y$ follows

$$
d Y_{t}=\left[a\left(Y_{t}\right)-b\left(Y_{t}\right)\left(\rho \lambda\left(Y_{t}\right)+\bar{\rho} \psi_{t}\right)\right] d t+b\left(Y_{t}\right) d \widetilde{W}_{t}^{Q},
$$

where $W^{Q}, \widetilde{W}^{Q}$ are $Q$-Brownian motions with correlation $\rho$. We write $\widetilde{W}^{Q}=\rho W^{Q}+\bar{\rho} W^{\perp, Q}$, where $\left(W^{Q}, W^{\perp, Q}\right)$ is a two-dimensional $Q$ Brownian motion given by $d W_{t}^{Q}=d W_{t}+\lambda\left(Y_{t}\right) d t, d W_{t}^{\perp, Q}=d W_{t}^{\perp}+\psi_{t} d t$. The traded asset $S$ is a local $Q$-martingale, and the $Q$-drift of $Y$ is arbitrary and parametrised by the integrand $\psi$ in (3).

The minimal martingale measure $Q^{M}$ of Föllmer and Schweizer (1991) corresponds to taking $\psi_{t}=0,0 \leq t \leq T$, and therefore $d Q^{M} / d P=$ : $Z_{T}^{M}:=\mathcal{E}(-\lambda \cdot W)_{T}$. We assume $\lambda(y)$ is such that $E Z_{T}^{M}=1$ so that $Q^{M}$ is a well-defined probability measure equivalent to $P$ on $\mathcal{F}_{T}$.

For $q \in(0,1]$, define measures $P^{(q, \psi)}$ as those with densities

$$
\frac{d P^{(q, \psi)}}{d P}=\mathcal{E}\left(-q \lambda \cdot W-q \psi \cdot W^{\perp}\right)_{T},
$$


where $\lambda, \psi$ are the same processes as in (3). The restriction of $P^{(q, \psi)}$ onto $\widetilde{\mathcal{F}}_{T}$ is $\widetilde{P}^{(q, \psi)}:=\left.P^{(q, \psi)}\right|_{\mathcal{F}_{T}}$ given by

$$
\frac{d \widetilde{P}^{(q, \psi)}}{d P}:=E\left[\frac{\left.d P^{(q, \psi}\right)}{d P} \mid \widetilde{\mathcal{F}}_{T}\right]=\mathcal{E}[-q(\rho \lambda+\bar{\rho} \psi) \cdot \widetilde{W}]_{T} .
$$

We sometimes refer to the measures $\widetilde{P}^{(q, \psi)}$ as distortion measures.

The "minimal" distortion measure, $\widetilde{P}^{M}:=\widetilde{P}^{(q, 0)}$, corresponding to $\psi=$ 0 , features prominently in this paper, and has density with respect to $P$ given by

$$
\frac{d \widetilde{P}^{M}}{d P}=\mathcal{E}(-q \rho \lambda \cdot \widetilde{W})_{T}
$$

corresponding to $\psi=0$ in (5) and (6). Note that our assumptions on $\lambda(y)$ imply that $\widetilde{P}^{M}$ is a probability measure equivalent to $P$ on $\widetilde{\mathcal{F}}_{T}$. Moreover, for $q=1, \widetilde{P}^{M}=\widetilde{Q}^{M}$, the projection of the minimal martingale measure $Q^{M}$ onto $\widetilde{\mathcal{F}}_{T}$.

For our purposes, the parameter $\lambda$ should be such that the function $H$ below is well-defined and suitably smooth. This function is the cornerstone of the distortion solution for the primal utility maximisation problem.

For $q \in(0,1]$, define $H:[0, T] \times \mathbb{R} \rightarrow \mathbb{R}^{+}$by

$$
H(t, y)=E^{\widetilde{P}^{M}}\left[\exp \left(-\frac{1}{2} q\left(1-q \rho^{2}\right) \int_{t}^{T} \lambda^{2}\left(Y_{s}\right) d s\right) \mid Y_{t}=y\right]
$$

This is clearly finite and bounded by $0<H(t, y) \leq 1$, for $q \in(0,1]$. We assume the parameter functions are such that $H \in C^{1,2}([0, T] \times \mathbb{R}) \cap$ $C([0, T] \times \mathbb{R})$. Under $\widetilde{P}^{M}$ the drift of $Y_{t}$ is $a\left(Y_{t}\right)-q \rho \lambda\left(Y_{t}\right) b\left(Y_{t}\right)$, and by the Feynman-Kac theorem $H(t, y)$ satisfies the linear PDE

$H_{t}+(a(y)-q \rho \lambda(y) b(y)) H_{y}+\frac{1}{2} b^{2}(y) H_{y y}-\frac{1}{2} q\left(1-q \rho^{2}\right) \lambda^{2}(y) H=0$,

with $H(T, y)=1$. Benth and Karlsen (2005) (see also Pham (2002)) consider the smoothness of the function $f:[0, T] \times \mathbb{R} \rightarrow \mathbb{R}$ defined by ${ }^{1}$

$$
f(t, y):=-\frac{1}{1-q \rho^{2}} \log H(t, y) .
$$

From the PDE (8) for $H, f(t, y)$ solves the semi-linear PDE

$$
f_{t}+(a(y)-q b \rho \lambda(y)) f_{y}+\frac{1}{2} b^{2}(y) f_{y y}-\frac{1}{2} b^{2}(y)\left(1-q \rho^{2}\right) f_{y}^{2}+\frac{1}{2} q \lambda^{2}(y)=0,
$$

\footnotetext{
1 Benth and Karlsen (2005) consider the case $q=1$, but their conclusions on the smoothness of $f$ are unaltered for $q \in(0,1]$.
} 
with $f(T, y)=0$. It is shown in Benth and Karlsen (2005) that $f$ (and hence $H$ ) is sufficiently smooth provided that the parameter functions $\lambda, a$ satisfy

$$
\begin{aligned}
& |a(y)| \leq C|y|,\left|a^{\prime}(y)\right| \leq C, \\
& |\lambda(y)| \leq C|y|,\left|\lambda^{\prime}(y)\right| \leq C,
\end{aligned}
$$

for a positive constant $C$ and $y \in \mathbb{R}$. Under these conditions there is a unique, quadratically growing classical solution of (10) with linearly growing derivative.

The conditions (11) are satisfied by the following version of the SteinStein (1991) model, considered in Benth and Karlsen (2005), with $\lambda(y)=$ $\lambda y$ for some constant $\lambda \neq 0, a(y)=m-\alpha y, b(y)=\beta$, with $m, \alpha, \beta$ positive constants:

$$
d S_{t}=Y_{t} S_{t}\left(\lambda Y_{t} d t+d W_{t}\right), d Y_{t}=\left(m-\alpha Y_{t}\right) d t+\beta d \widetilde{W}_{t} .
$$

Although (11) is not satisfied by the Heston (1993) model, it turns out that the function $H$ is also smooth in this case, as shown in Benth and Karlsen (2005), who consider a version of the Heston model with $\lambda(y)=\lambda y$ for some constant $\lambda \neq 0, a(y)=(m / y)-\alpha y, b(y)=\beta$, with $m, \alpha, \beta$ positive constants:

$$
d S_{t}=Y_{t} S_{t}\left(\lambda Y_{t} d t+d W_{t}\right), d Y_{t}=\left(\frac{m}{Y_{t}}-\alpha Y_{t}\right) d t+\beta d \tilde{W}_{t},
$$

with $m \geq \frac{1}{2} \beta^{2}$ ensuring that the squared volatility $Y_{t}^{2}=: V_{t}$ is positive.

\subsection{The primal problem}

In the primal utility maximisation problem, an agent trades a self-financing portfolio involving the traded asset, so that the portfolio wealth process $X^{\pi} \equiv X:=\left(X_{t}\right)_{0 \leq t \leq T}$ satisfies

$$
d X_{t}=\pi_{t} Y_{t}\left(\lambda\left(Y_{t}\right) d t+d W_{t}\right),
$$

where $\pi:=\left(\pi_{t}\right)_{0 \leq t \leq T}$ is the wealth invested in the stock, representing the agent's trading strategy. A trading strategy is an adapted process $\pi$ satisfying $\int_{0}^{T} Y_{t}^{2} \pi_{t}^{2} d t<\infty$ almost surely. Let $\mathcal{A}_{0}$ denote the set of trading strategies.

The agent has risk preferences expressed via a concave utility function $U(x)$. We consider the two cases:

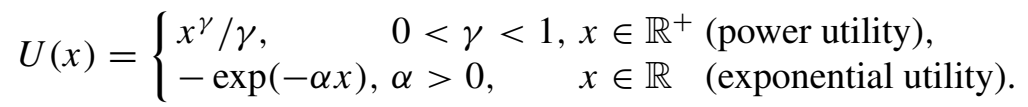


The objective is to maximise expected utility of terminal wealth at time $T$, given initial capital $X_{0}=x$ :

$$
u(x):=\sup _{\pi \in \mathcal{A}} E U\left(X_{T}\right),
$$

where $\mathcal{A}$ denotes a set of admissible trading strategies.

For power utility, an admissible trading strategy is one whose wealth process satisfies $X \geq 0$, a.s.

For exponential utility, let $\mathcal{M}_{f}$ denote the set of martingale measures $Q$ with finite relative entropy with respect to $P$. Then, defining $\Delta:=\pi / S$ as the adapted $S$-integrable process for the number of shares held, we follow Becherer (2004) and take the space of permitted strategies as

$$
\mathcal{A}=\left\{\Delta:(\Delta \cdot S) \text { is a }(Q, \mathbb{F}) \text {-martingale for all } Q \in \mathcal{M}_{f}\right\},
$$

where $(\Delta \cdot S)_{t}=\int_{0}^{t} \Delta_{s} d S_{s}$ is the gain from trading over $[0, t], t \in[0, T]$. Other choices for $\mathcal{A}$ are possible. For instance, one may follow Schachermayer (2001) and consider strategies with wealth bounded from below, and then maximise over the $L^{1}(P)$-closure of the set of all random variables $U(\Gamma)$ such that $\Gamma$ can be super-hedged by some trading strategy. The good news is that these choices lead to the same solution for the dual to the primal utility maximisation problem (Delbaen et al. (2002), Kabanov and Stricker (2002), Schachermayer (2001)).

For a starting time $t \in[0, T]$ the agent's maximum utility, or primal value function, is

$$
u(t, x, y):=\sup _{\pi \in \mathcal{A}} E\left[U\left(X_{t}\right) \mid X_{t}=x, Y_{t}=y\right],
$$

with $u(0, x, y) \equiv u(x)$ given in (16).

\subsection{The dual problem}

The dual problem to (16) is defined by

$$
v(\eta):=\inf _{Q \in \mathcal{M}} E V\left(\eta \frac{d Q}{d P}\right), \eta>0,
$$

where $V: \mathbb{R}^{+} \rightarrow \mathbb{R}$ is the convex conjugate of the utility function $U(\cdot)$ :

$$
V(\eta):=\sup _{x \in \operatorname{dom}(U)}(U(x)-x \eta), \eta>0 .
$$

For the utility functions in (15), $V$ is given by

$$
V(\eta)=\left\{\begin{array}{lrl}
-\left(\eta^{q} / q\right), & q=-\left(\frac{\gamma}{1-\gamma}\right), & U(x)=x^{\gamma} / \gamma, \\
(\eta / \alpha)(\log (\eta / \alpha)-1), & U(x)=-e^{-\alpha x} .
\end{array}\right.
$$


Denote the optimiser in (19) by $Q^{*}$. Then, for power utility, $Q^{*}=Q^{(q)}$ maximising (for $q \in(0,1)$ ) the functional

$$
d_{q}(Q, P):=E\left[\left(\frac{d Q}{d P}\right)^{q}\right], 0<q<1,
$$

over $Q \in \mathcal{M}$, with $q=-\gamma /(1-\gamma)$. The measure $Q^{(q)}$ is sometimes called the $q$-optimal measure, and is studied by Hobson (2004) for $q \geq 1$.

For exponential utility, (20) implies that the dual optimiser is the minimal entropy measure $Q^{E}$, which minimises the relative entropy

$$
d_{1}(Q, P):=E\left[\frac{d Q}{d P} \log \frac{d Q}{d P}\right]
$$

over $Q \in \mathcal{M}_{f}$. As Hobson (2004) has shown, the minimal entropy measure can be viewed as the $q$-optimal measure for $q=1, Q^{E}=Q^{(1)}$.

A well-known body of work (Karatzas et al. (1991), Kramkov and Schachermayer (1999), Goll and Rüschendorf (2001), Bellini and Frittelli (2002), Delbaen et al. (2002), Frittelli (2000), Owen (2002)) has established the fundamental duality relations between $u(x)$ and $v(\eta)$. The value functions $u(x), v(\eta)$ are conjugate, inheriting these properties from $U, V$, and the optimal terminal wealth in (16), $X_{T}^{*}$, is related to the optimal dual measure $Q^{*}$ by

$$
U^{\prime}\left(X_{T}^{*}\right)=u^{\prime}(x) \frac{d Q^{*}}{d P} .
$$

For an initial time $t \in[0, T]$ the dual value function is defined by the dual stochastic control problem

$$
v(t, \eta, y):=\inf _{\psi \in \Psi} E\left[V\left(\eta \frac{Z_{T}}{Z_{t}}\right) \mid Y_{t}=y\right]
$$

with $Z_{t}=\mathcal{E}\left(-\lambda \cdot W-\psi \cdot W^{\perp}\right), 0 \leq t \leq T$, defined in (3), and where $\Psi$ denotes the set of processes $\psi$ satisfying $\int_{0}^{T} \psi_{t}^{2} d t<\infty$. We have $v(0, \eta, y) \equiv v(\eta)$, and the dual optimiser $Q^{*}$ has density with respect to $P$ given by

$$
\frac{d Q^{*}}{d P}=\mathcal{E}\left(-\lambda \cdot W-\xi \cdot W^{\perp}\right)_{T}
$$

for an optimal process $\psi^{*} \equiv \xi:=\left(\xi_{t}\right)_{0 \leq t \leq T}$ achieving the infimum in (22). The value functions $u(t, x, y)$ and $v(t, \eta, y)$ are conjugate, in the same manner as their time 0 counterparts $u(x)$ and $v(\eta)$. 


\section{The distortion solution to the primal problem}

The following solution for the primal value function was established by various authors under varying conditions. Under global Lipschitz and linear growth conditions on the parameter functions, we refer the reader to Zariphopoulou (2001) for proofs based on viscosity solution techniques. Under further conditions (boundedness and Hölder continuity of the parameter functions, and uniform ellipticity of $b(y))$, Zariphopoulou (2001) shows that the value function is in fact a smooth solution of the associated Hamilton-Jacobi-Bellman (HJB) equation. Tehranchi (2004) proves the distortion representation below in a non-Markovian scenario with boundedness conditions on the market parameters. Below we use formal PDE arguments, assuming the value function has sufficient regularity and smoothness. This is true if the parameters satisfy (11), and for the versions of the Stein-Stein and Heston models given in (12) and (13). We verify optimality by appealing to subsequent results, in which we establish optimality of the candidate optimal measure (in the dual problem) implied by the distortion solution of the primal problem.

Proposition 1 (Distortion Power Solution). For $0<q \leq 1$, the value function (18) is given by

$$
u(t, x, y)=U(x)(H(t, y))^{\delta},
$$

where

$$
\delta=\frac{1}{1-q \rho^{2}},
$$

with $q=1$ for exponential utility, $q=-\gamma /(1-\gamma)$ for power utility, and $H(t, y)$ given in (7).

Proof. The HJB equation for $u(t, x, y)$ is

$$
\max _{\pi}\left(u_{t}+L^{Y} u+\pi y \lambda(y) u_{x}+\frac{1}{2} \pi^{2} y^{2} u_{x x}+\pi \rho y b(y) u_{x y}\right)=0,
$$

where $\mathcal{L}^{Y}$ is the generator of the process $Y$ :

$$
\mathcal{L}^{Y} u:=a(y) u_{y}+\frac{1}{2} b^{2}(y) u_{y y} .
$$

Performing the maximisation then gives the optimal (Markov) control $\pi^{*}(t, x, y)$ as

$$
\pi^{*}(t, x, y)=-\frac{\left(\lambda(y) u_{x}+\rho b(y) u_{x y}\right)}{y u_{x x}} .
$$


The optimal trading strategy $\pi^{*}:=\left(\pi_{t}^{*}\right)_{0 \leq t \leq T}$ is given by $\pi_{t}^{*}=$ $\pi^{*}\left(t, X_{t}^{*}, Y_{t}\right)$, where $X_{t}^{*}$ is the wealth process (14) with $\pi_{t}=\pi_{t}^{*}$. Inserting (25) into the Bellman equation gives

$$
u_{t}+a(y) u_{y}+\frac{1}{2} b^{2}(y) u_{y y}-\frac{1}{2 u_{x x}}\left(\lambda(y) u_{x}+\rho b(y) u_{x y}\right)^{2}=0,
$$

with $u(T, x, y)=U(x)$. Seek a separable solution to (26) of the form (23), in turn for power and exponential preferences. Then it is straightforward to verify that $\delta$ as in (24) and $q$ as given in the proposition results in $H$ satisfying the linear PDE (8), and the form of $u$ is proved. A verification theorem establishes that the solution is indeed the value function. In fact, we show below that the proposed solution is the value function by appealing to duality, demonstrating that the candidate optimal measure for the dual problem, implied by the above solution for the primal problem, is in fact the dual optimiser. This is achieved by showing that the representation equation of Hobson (2004) for the dual optimiser is satisfied.

\section{Representations for the dual optimiser}

We begin with a simple lemma, establishing the relation between the dual value function (and hence the dual optimiser $Q^{*}$ ), and the function $H$ appearing in the distortion solution of the primal problem.

Lemma 1. The dual optimiser $Q^{*}$ achieving the infimum

$$
v(\eta):=\inf _{Q \in \mathcal{M}} E V\left(\eta \frac{d Q}{d P}\right)
$$

satisfies

$$
v(\eta) \equiv E V\left(\eta \frac{d Q^{*}}{d P}\right)=H^{\delta} V\left(\frac{\eta}{H^{\delta}}\right),
$$

where $H \equiv H\left(0, Y_{0}\right)$ is given in (7) and $\delta$ is given in (24).

Proof. The dual value function $v(\eta)$ is the convex conjugate of $u(x)$ :

$$
v(\eta)=\sup _{x \in \operatorname{dom}(U)}[u(x)-x \eta]
$$

which implies that, for any $\eta>0$, the value of $x$ achieving the above supremum is $x^{*} \equiv x^{*}(\eta)$ satisfying $u^{\prime}\left(x^{*}\right)=\eta$. Using (23) at $t=0$, and writing $H \equiv H\left(0, Y_{0}\right)$, we see that this condition becomes $U^{\prime}\left(x^{*}\right) H^{\delta}=\eta$, 
or $x^{*}=I\left(\eta / H^{\delta}\right)$, where $I(\cdot)=\left(U^{\prime}(\cdot)\right)^{-1}$ is the inverse of the gradient of the utility function $U$. Hence

$$
\begin{aligned}
v(\eta) & =u\left(x^{*}\right)-x^{*} \eta \\
& =H^{\delta}\left[U\left(I\left(\eta / H^{\delta}\right)\right)-\left(\eta / H^{\delta}\right) I\left(\eta / H^{\delta}\right)\right],
\end{aligned}
$$

and the result follows from the identity $V(\eta)=U(I(\eta))-\eta I(\eta)$.

Written explicitly, Lemma 1 states that $Q^{*}$ and $\widetilde{P}^{M}$ are related by:

- For exponential utility, with $q=1$ (so that $\widetilde{P}^{M}=\widetilde{Q}^{M}$ ),

$$
\begin{aligned}
E\left[\frac{d Q^{E}}{d P} \log \frac{d Q^{E}}{d P}\right] & =-\frac{1}{1-\rho^{2}} \log E^{\widetilde{Q}^{M}} e^{-\frac{1}{2}\left(1-\rho^{2}\right) K_{T}} \\
& =-\frac{1}{1-\rho^{2}} \log H\left(0, Y_{0}\right),
\end{aligned}
$$

where $Q^{*}=Q^{E}=Q^{(1)}$ is the minimal entropy measure. Independently, Stoikov and Zariphopoulou (2004) (Corollaries 2.1 and 3.1) have also derived this result.

- For power utility, with $q=-\gamma /(1-\gamma)$,

$$
\begin{aligned}
E\left[\left(\frac{d Q^{(q)}}{d P}\right)^{q}\right] & =\left(E^{\widetilde{P}^{M}} e^{-\frac{1}{2} q\left(1-q \rho^{2}\right) K_{T}}\right)^{(1-q) /\left(1-q \rho^{2}\right)} \\
& =\left[H\left(0, Y_{0}\right)\right]^{(1-q) /\left(1-q \rho^{2}\right)}
\end{aligned}
$$

where $Q^{*}=Q^{(q)}$ is the $q$-optimal measure. This result, written in a different form, appears in Hobson (2004).

Naturally, an analogous relationship to (27) can be derived between the dual value function $v(t, \eta, y)$ for initial time $t \in[0, T]$ and $H(t, y)$. This yields that the dual value function $v(t, \eta, y)$ is related to $H(t, y)$ and $\delta=$ $1 /\left(1-q \rho^{2}\right)$ by

$$
v(t, \eta, y)= \begin{cases}V(\eta)(H(t, y))^{\delta(1-q)}, & 0<q<1, \\ V(\eta)-(\eta / \alpha) \log \left((H(t, y))^{\delta}\right), & q=1 .\end{cases}
$$

We now attack the dual problem (22) directly via dynamic programming and the associated HJB equation. Fusing this with (30) gives the following representation for the optimiser $Q^{*}$.

Theorem 1. The dual optimiser $Q^{*}$ has density given by

$$
\frac{d Q^{*}}{d P}=\mathcal{E}\left(-\lambda \cdot W-\xi \cdot W^{\perp}\right)_{T}
$$


with

$$
\xi_{t}=-\frac{\sqrt{1-\rho^{2}}}{1-q \rho^{2}} b\left(Y_{t}\right) \frac{\partial}{\partial y} \log H\left(t, Y_{t}\right), t \in[0, T], 0<q \leq 1,
$$

and where $H(t, y)$ is given in (7).

Proof. From the definition (22) and the $P$-dynamics (2) of $Y$ and (4) of $Z$, the Bellman equation for $v$ is

$$
\inf _{\psi}\left[v_{t}+\mathcal{L}^{Y} v+\frac{1}{2}\left(\lambda^{2}(y)+\psi^{2}\right) \eta^{2} v_{\eta \eta}-(\rho \lambda(y)+\bar{\rho} \psi) b(y) \eta v_{\eta y}\right]=0 .
$$

Performing the minimisation gives the optimal value of $\psi$ in feedback form as $\psi^{*} \equiv \xi$ given by

$$
\xi(t, \eta, y)=\frac{\bar{\rho} b(y)}{\eta} \frac{v_{\eta y}(t, \eta, y)}{v_{\eta \eta}(t, \eta, y)} .
$$

Using (30), in turn for $q=1$ and for $0<q<1$, we see that (32) loses all dependence on $\eta$ and reduces to

$$
\xi(t, y)=-\frac{\sqrt{1-\rho^{2}}}{1-q \rho^{2}} b(y) \frac{H_{y}(t, y)}{H(t, y)} .
$$

The optimal control process $\xi:=\left(\xi_{t}\right)_{0 \leq t \leq T}$ is given by $\xi_{t}=\xi\left(t, Y_{t}\right)$. The optimality of the proposed control follows from a verification theorem, or alternatively from the fact that $\xi$ given in (33) does indeed solve the representation equation of Hobson (2004) which identifies the optimal dual measure, and we demonstrate this fact below.

\subsection{A representation equation for the dual minimiser}

Theorem 1 identifies a candidate optimal dual measure $Q^{*}$ and associated optimal dual control process $\xi$. To verify that these candidates are indeed optimal, we show that they satisfy a martingale representation equation of Hobson (2004) for the $q$-optimal measure $Q^{(q)} \in \mathcal{M}(q \in \mathbb{R})$ in stochastic volatility models. Recall that we identify the $q$-optimal measure with the optimal dual measure $Q^{*}$, with $0<q<1$ corresponding to power utility, and $q=1$ corresponding to exponential utility (in which case Hobson's result reduces to a representation equation of Rheinländer (2005)). The case $q=0$ corresponds to logarithmic utility, and is not analysed here. This case is somewhat degenerate, in that the optimal dual measure is the minimal measure $Q^{M}$ (as the reader can easily verify), in accordance with a general result of Schweizer (1999). 
The representation equation was established in Hobson (2004) for $q \geq 1$, and it was pointed out there and in Henderson et al. (2005) that the result extends to $q<1$. We verify this claim for the case $0<q<1$ after stating the result, and we give integrability conditions under which the representation equation does indeed identify the dual optimiser $Q^{*}$.

Proposition 2 (Hobson (2004)). Suppose there are previsible processes $v$ and $\xi$ and a finite constant $c$ such that

$$
\frac{1}{2} q K_{T}=M_{T}+\frac{1}{2}(1-q)[M]_{T}+L_{T}+\frac{1}{2}[L]_{T}+c,
$$

where

$$
M_{t}:=\int_{0}^{t} v_{s}\left(d W_{s}+q \lambda_{s} d s\right), L_{t}:=\int_{0}^{t} \xi_{s} d W_{s}^{\perp} .
$$

Then, for $q \in(0,1]$, the q-optimal measure $Q^{(q)}$ (equivalently the dual optimiser $\left.Q^{*}\right)$ is given by

$$
\frac{d Q^{*}}{d P}=\mathcal{E}\left(-\lambda \cdot W-\xi \cdot W^{\perp}\right)_{T}
$$

and the constant $c$ is given by

$$
\begin{gathered}
c=E\left[\frac{d Q^{*}}{d P} \log \frac{d Q^{*}}{d P}\right], \quad q=1, \\
e^{-(1-q) c}=E\left[\left(\frac{d Q^{*}}{d P}\right)^{q}\right], 0<q<1,
\end{gathered}
$$

provided that the local martingale $Z^{*}:=\mathcal{E}\left(-\lambda \cdot W-\xi \cdot W^{\perp}\right)$ is a true martingale, and provided that the following integrability conditions are satisfied:

- for $q=1:(v-\lambda) \cdot W^{Q}$ is a $Q$-martingale for all $Q \in \mathcal{M}$;

- for $0<q<1$, the local $Q$-martingale $\mathcal{E}\left[-(1-q)(v-\lambda) \cdot W^{Q}\right]$ is a true $Q$-martingale for all $Q \in \mathcal{M}$.

We first show that a solution to (34) can be found in terms of our candidate dual control process $\xi$ of Theorem 1 .

Proposition 3. Proposition 2 holds for the triple $(\nu, \xi, c)$ given by

$$
\bar{\rho} v_{t}-\rho \xi_{t}=0,
$$

with $\xi_{t}$ given by (31): 


$$
\xi_{t}=-\frac{\sqrt{1-\rho^{2}}}{1-q \rho^{2}} b\left(Y_{t}\right) \frac{\partial}{\partial y} \log H\left(t, Y_{t}\right)
$$

and

$$
c=-\frac{1}{1-q \rho^{2}} \log H\left(0, Y_{0}\right) .
$$

Proof. First note that with $c$ given by (39), then (36) and (37) are implied by (28) and (29).

For $q \in(0,1]$, define the function $f:[0, T] \times \mathbb{R} \rightarrow \mathbb{R}$ by (9):

$$
f(t, y):=-\frac{1}{1-q \rho^{2}} \log H(t, y),
$$

so that $f$ solves the semi-linear $\operatorname{PDE}(10)$. With $\xi$ as in the proposition, $M, L$ are given by

$$
\begin{aligned}
M_{t} & =\rho \int_{0}^{t} b\left(Y_{s}\right) f_{y}\left(s, Y_{s}\right)\left(d W_{s}+q \lambda\left(Y_{s}\right) d s\right), \\
L_{t} & =\bar{\rho} \int_{0}^{t} b\left(Y_{s}\right) f_{y}\left(s, Y_{s}\right) d W_{s}^{\perp} .
\end{aligned}
$$

With $\widetilde{W}=\rho W+\bar{\rho} W^{\perp}$, a $(P, \widetilde{\mathbb{F}})$ Brownian motion, a direct computation gives

$$
\begin{aligned}
& M_{T}+\frac{1}{2}(1-q)[M]_{T}+L_{T}+\frac{1}{2}[L]_{T} \\
= & \int_{0}^{T} b\left(Y_{t}\right) f_{y}\left(t, Y_{t}\right) d \widetilde{W}_{t} \\
+ & \int_{0}^{T}\left(q \rho \lambda\left(Y_{t}\right) b\left(Y_{t}\right) f_{y}\left(t, Y_{t}\right)+\frac{1}{2}\left(1-q \rho^{2}\right) b^{2}\left(Y_{t}\right) f_{y}^{2}\left(t, Y_{t}\right)\right) d t \\
= & \frac{1}{2} q K_{T}+\int_{0}^{T}\left\{\left(f_{t}\left(t, Y_{t}\right)+\mathcal{L}^{Y} f\left(t, Y_{t}\right)\right) d t+b\left(Y_{t}\right) f_{y}\left(t, Y_{t}\right) d \widetilde{W}_{t}\right\} \\
= & \frac{1}{2} q K_{T}+\int_{0}^{T} d f\left(t, Y_{t}\right) \\
= & =\frac{1}{2} q K_{T}-f\left(0, Y_{0}\right) \\
= & \frac{1}{2} q K_{T}+\frac{1}{1-q \rho^{2}} \log H\left(0, Y_{0}\right) \\
= & \frac{1}{2} q K_{T}-c,
\end{aligned}
$$

where we have used the PDE (10) satisfied by $f$ and the Itô formula. This establishes that the representation equation (34) is satisfied. 
It remains to show that the candidate measure $Q^{\star}$ is indeed optimal provided the relevant integrability conditions hold. We shall need the following simple lemma, an adaptation of Lemma 3.4 in Hobson (2004) and easily proven by similar methods to those in Hobson (2004).

Lemma 2. For any $\alpha \in(0,1)$, adapted integrand $\phi$ and Brownian motion $B$, we have

$$
E\left[\left(\mathcal{E}(\phi \cdot B)_{T}\right)^{\alpha}\right] \leq 1, E\left[\int_{0}^{T} \phi_{t} d B_{t}+\frac{1}{2} \phi_{t}^{2} d t\right] \geq 0 .
$$

Now, with $(v, \xi, c)$ as in the proposition, the candidate dual optimiser $Q^{*}$ is defined by (35). Let $Q$ be any martingale measure, with density given by (3) for some process $\psi$ satisfying $\int_{0}^{T} \psi_{t}^{2} d t<\infty P$-almost surely. Defining the two-dimensional $Q$-Brownian motion $\left(W^{Q}, W^{\perp, Q}\right)$ by $d W_{t}^{Q}=d W_{t}+$ $\lambda\left(Y_{t}\right) d t, d W_{t}^{\perp, Q}=d W_{t}^{\perp}+\psi_{t} d t$, we may rewrite the representation equation (34) in terms of $\left(W^{Q}, W^{\perp, Q}\right)$ as

$$
\begin{aligned}
\frac{1}{2} q K_{T} & =\left(v \cdot W^{Q}\right)_{T}+(1-q) \int_{0}^{T}\left(\frac{1}{2} v_{t}^{2}-v_{t} \lambda_{t}\right) d t+\left(\xi \cdot W^{\perp, Q}\right)_{T} \\
& +\int_{0}^{T}\left(\frac{1}{2} \xi_{t}^{2}-\xi_{t} \psi_{t}\right) d t
\end{aligned}
$$

Now write $\log Z_{T} \equiv \log (d Q / d P)$ in terms of $\left(W^{Q}, W^{\perp, Q}\right)$ as

$$
\log Z_{T}=-\left(\lambda \cdot W^{Q}\right)_{T}+\frac{1}{2} K_{T}-\left(\psi \cdot W^{\perp, Q}\right)_{T}+\frac{1}{2} \int_{0}^{T} \psi_{t}^{2} d t .
$$

Use the trivial decomposition $\frac{1}{2} K_{T}=\frac{1}{2} q K_{T}+\frac{1}{2}(1-q) K_{T}$ and (40) to obtain

$$
\begin{aligned}
\log Z_{T} & =\left((\nu-\lambda) \cdot W^{Q}\right)_{T}+\left((\xi-\psi) \cdot W^{\perp, Q}\right)_{T} \\
& +\frac{1}{2} \int_{0}^{T}\left\{(1-q)\left(v_{t}-\lambda_{t}\right)^{2}+\left(\xi_{t}-\psi_{t}\right)^{2}\right\} d t+c .
\end{aligned}
$$

Then, for $0<q<1$, we have

$$
\begin{aligned}
& d_{q}(Q, P)=E\left[Z_{T}^{q}\right]=E^{Q}\left[Z_{T}^{-(1-q)}\right] \\
= & E^{Q}\left[\mathcal{E}\left[-(1-q)(v-\lambda) \cdot W^{Q}\right]_{T}\left\{\mathcal{E}\left[(\xi-\psi) \cdot W^{\perp, Q}\right]_{T}\right\}^{1-q}\right] e^{-(1-q) c} .
\end{aligned}
$$

If $\Lambda:=\mathcal{E}\left[-(1-q)(\nu-\lambda) \cdot W^{Q}\right]$ is a true $Q$-martingale for all $Q \in \mathcal{M}$, then it can be used to define a measure change to a measure $R$, by $d R / d Q:=\Lambda_{T}$. After this measure change, $W^{Q, \perp}$ is still a Brownian motion, which we denote by $W^{R, \perp}$, and we obtain

$$
d_{q}(Q, P)=E^{R}\left[\left\{\mathcal{E}\left[(\xi-\psi) \cdot W^{R, \perp}\right]_{T}\right\}^{1-q}\right] e^{-(1-q) c} \leq e^{-(1-q) c},
$$


the inequality following from Lemma 2 , with equality if $\psi=\xi$, so that $d_{q}(Q, P)$ is maximised over all $Q \in \mathcal{M}$ by this choice.

For the case $q=1$, we have, by the same analysis as above,

$$
\begin{aligned}
& d_{1}(Q, P)=E\left[Z_{T} \log Z_{T}\right]=E^{Q} \log Z_{T}=E^{Q}\left[(\nu-\lambda) \cdot W^{Q}\right]_{T} \\
& +E^{Q}\left[\left((\xi-\psi) \cdot W^{\perp, Q}\right)_{T}+\frac{1}{2} \int_{0}^{T}\left(\xi_{t}-\psi_{t}\right)^{2} d t\right]+c .
\end{aligned}
$$

If $(v-\lambda) \cdot W^{Q}$ is a $q$-martingale the first term in the last line vanishes and we have

$$
d_{1}(Q, P)=E^{Q}\left[\left((\xi-\psi) \cdot W^{\perp, Q}\right)_{T}+\frac{1}{2} \int_{0}^{T}\left(\xi_{t}-\psi_{t}\right)^{2} d t\right]+c \geq c,
$$

the inequality following from Lemma 2 , with equality if $\psi=\xi$, so that $d_{1}(Q, P)$ is minimised over all $Q \in \mathcal{M}$ by this choice.

This establishes the optimality of the proposed candidate dual optimiser, and by duality, the optimality of the distortion solution for the primal problem.

An immediate corollary of the representation equation (34) is the following representation of the likelihood ratio $d Q^{*} / d P$, given in Hobson (2004) for $q \geq 1$ and extended here to $0<q \leq 1$.

Corollary 1. The dual optimiser has Radon-Nikodym derivative given in terms of the traded asset price $S$ by

$$
\log \frac{d Q^{*}}{d P}=c+\int_{0}^{T} \theta_{t} d S_{t}+\frac{1}{2}(1-q) \int_{0}^{T} \theta_{t}^{2} d[S]_{t},
$$

where

$$
\theta_{t}:=\frac{\nu_{t}-\lambda\left(Y_{t}\right)}{Y_{t} S_{t}}, 0 \leq t \leq T .
$$

Proof. This follows immediately on using (41) with $\psi=\xi$.

\subsection{Relation between optimal portfolio and dual optimiser}

The process $\theta$ in Corollary 1 is closely related to the optimal trading strategy, as we now show. For a utility function $U(x)$, denote the Arrow-Pratt measure of risk aversion by $A(x):=-U^{\prime \prime}(x) / U^{\prime}(x)$. Then we have the following relation between the primal and dual feedback control functions. 
Proposition 4. The optimal portfolio process $\pi^{*}$ is given by

$$
\pi_{t}^{*}=-\frac{\theta_{t} S_{t}}{A\left(X_{t}^{*}\right)}
$$

where $\theta_{t}$ is the process in (42), and $X_{t}^{*}$ is the optimal wealth process (14) with $\pi_{t}=\pi_{t}^{*}$.

Proof. Use the distortion solution (23) in (25) to give the optimal portfolio feedback control $\pi^{*}(t, x, y)$ as

$$
\begin{aligned}
\pi^{*}(t, x, y) & =\frac{1}{y A(x)}\left(\lambda(y)+\delta \rho b(y) \frac{H_{y}(t, y)}{H(t, y)}\right) \\
& =-\frac{1}{y A(x)}\left(\left(\frac{\rho}{\bar{\rho}}\right) \xi(t, y)-\lambda(y)\right),
\end{aligned}
$$

the last equality following from (33). Then (43) follows from $\pi_{t}^{*}=$ $\pi^{*}\left(t, X_{t}^{*}, Y_{t}\right)$, along with (38) and the expression (42) for $\theta$.

Remark 1. Note that for exponential utility, with $A(x)=\alpha$, the condition that $(v-\lambda) \cdot W^{Q}$ is a $Q$-martingale becomes, on using (43), that $\Delta^{*} \cdot S$ is a $Q$-martingale, where $\Delta^{*}=\pi^{*} / S$ is the optimal number of shares. This is in accordance with the definition of the admissible trading strategies for the exponential utility case, given in (17).

Proposition 4 is an example of the classical relation (21) between the dual optimiser and the optimal terminal wealth:

Corollary 2. The relation (43) implies (21).

Proof. We prove the result for power utility, $0<q<1$. The proof for exponential preferences follows the same lines. Using (43) in (14), the optimal wealth process follows

$$
d X_{t}^{*}=-\frac{\theta_{t}}{A\left(X_{t}^{*}\right)} d S_{t}
$$

For power utility, $A(x)=1 /(x(1-q))$. Using this and applying the Itô formula gives the logarithm of optimal terminal wealth, given $X_{0}=x$, as

$$
\log X_{T}^{*}=\log x-(1-q)\left(\int_{0}^{T} \theta_{t} d S_{t}+\frac{1}{2}(1-q) \int_{0}^{T} \theta_{t}^{2} d[S]_{t}\right) .
$$

Using Corollary 1 this may be rearranged to

$$
X_{T}^{*}=x e^{(1-q) c}\left(\frac{d Q^{*}}{d P}\right)^{-(1-q)} .
$$


Using (39) to eliminate $c$ in favour of $H\left(0, Y_{0}\right)$ we get

$$
X_{T}^{*}=x\left[H\left(0, Y_{0}\right)\right]^{-(1-q) /\left(1-q \rho^{2}\right)}\left(\frac{d Q^{*}}{d P}\right)^{-(1-q)} .
$$

For power utility, $U^{\prime}(x)=x^{-1 /(1-q)}$. We also have $u(x)=U(x)$ $\times\left[H\left(0, Y_{0}\right)\right]^{1 /\left(1-q \rho^{2}\right)}$ from the distortion solution. Using these facts we convert (44) to

$$
U^{\prime}\left(X_{T}^{*}\right)=u^{\prime}(x) \frac{d Q^{*}}{d P}
$$

\subsection{A novel representation of the dual control problem}

Define the functional $C^{q, \psi}$ by

$$
C^{q, \psi}:= \begin{cases}\exp \left\{-\frac{1}{2} q(1-q) \int_{0}^{T}\left(\lambda^{2}\left(Y_{t}\right)+\psi_{t}^{2}\right) d t\right\}, & 0<q<1, \\ \frac{1}{2} \int_{0}^{T}\left(\lambda^{2}\left(Y_{t}\right)+\psi_{t}^{2}\right) d t, & q=1 .\end{cases}
$$

Theorem 2. For $0<q \leq 1$, the dual problem is the stochastic control problem to minimise (maximise, for $q \in(0,1)$ )

$$
d_{q}(Q, P)=E^{P^{(q, \psi)}} C^{q, \psi},
$$

over processes $\psi \in \Psi$ satisfying $\int_{0}^{T} \psi_{t}^{2} d t<\infty$. The optimal control $\psi^{*}=$ : $\xi$ satisfies

$$
\Phi^{(q)}\left(E^{P^{(q, \xi)}} C^{q, \xi}\right)=E^{P^{q, 0}} \Phi^{(q)}\left(C^{q, 0}\right),
$$

where $\Phi^{(q)}(\cdot)$ is given by

$$
\Phi^{(q)}(x)= \begin{cases}x^{\left(1-q \rho^{2}\right) /(1-q)}, & 0<q<1, \\ \exp \left\{\left(1-\rho^{2}\right) x\right\}, & q=1 .\end{cases}
$$

Proof. We prove the result for $0<q<1$ (power utility). The case $q=1$ is proved similarly.

For a martingale measure $Q \in \mathcal{M}$ we have, with $Z_{T} \equiv d Q / d P=$ $\mathcal{E}\left(-\lambda \cdot W-\psi \cdot W^{\perp}\right)_{T}$,

$$
d_{q}(Q, P)=E\left[Z_{T}^{q}\right]=E^{P^{(q, \psi)}}\left[\frac{Z_{T}^{q}}{d P^{(q, \psi)} / d P}\right]=E^{P^{(q, \psi)}} C^{q, \psi},
$$

so that

$$
d_{q}\left(Q^{*}, P\right)=\sup _{\psi \in \Psi} E^{P^{(q, \psi)}} C^{q, \psi}=E^{P^{(q, \xi)}} C^{q, \xi} .
$$


Using (29) to substitute for $d_{q}\left(Q^{*}, P\right)$, we obtain

$$
\begin{aligned}
\sup _{\psi \in \Psi} E^{P^{(q, \psi)}} C^{q, \psi} & =\left(E^{\widetilde{P}^{M}} e^{-\frac{1}{2} q\left(1-q \rho^{2}\right) K_{T}}\right)^{(1-q) /\left(1-q \rho^{2}\right)} \\
& =\left\{E^{\widetilde{P}^{(q, 0)}}\left[\left(C^{q, 0}\right)^{\left(1-q \rho^{2}\right) /(1-q)}\right]\right\}^{(1-q) /\left(1-q \rho^{2}\right)},
\end{aligned}
$$

where we have used $\widetilde{P}^{M}=\widetilde{P}^{(q, 0)}$. Since $C^{q, 0}$ is $\widetilde{\mathcal{F}}_{T}$-measurable we can replace $\widetilde{P}^{(q, 0)}$ by $P^{(q, 0)}$ in the expectation on the right hand side, and the required formula follows. We know that the above supremum is attained from the proof of Proposition 3, and this completes the proof.

\subsection{Esscher transform relations}

Theorem 3. For $q \in(0,1]$, define the process $\chi:=\left(\chi_{t}\right)_{0 \leq t \leq T}$ by

$$
\chi_{t}:=\frac{1-q \rho^{2}}{q\left(1-\rho^{2}\right)} \xi_{t}
$$

where $\xi$ is the optimal dual control process (31). Then we have the Esscher transform relation

$$
\frac{d \widetilde{P}^{(q, \chi)}}{d \widetilde{P}^{M}}=\frac{\exp \left(-\frac{1}{2} q\left(1-q \rho^{2}\right) K_{T}\right)}{E^{\widetilde{P}^{M}} \exp \left(-\frac{1}{2} q\left(1-q \rho^{2}\right) K_{T}\right)} .
$$

Proof. We use the representation equation (34) and Proposition 3. Introduce the orthogonal Brownian motions

$$
\widetilde{W}:=\rho W+\bar{\rho} W^{\perp}, \widetilde{W}^{\perp}:=\bar{\rho} W-\rho W^{\perp},
$$

so that $\widetilde{W}$ is $\widetilde{\mathbb{F}}$-adapted and $\widetilde{W}^{\perp}$ is independent of $\widetilde{\mathbb{F}}$. We write the representation equation (34) in terms of $\widetilde{W}, \widetilde{W}^{\perp}$, and also use (38) and (39) to substitute for $v, c$. This yields

$$
\begin{aligned}
\frac{1}{2} q K_{T} & =\frac{1}{\bar{\rho}} \int_{0}^{T} \xi_{t} d \widetilde{W}_{t}+\frac{1}{\bar{\rho}^{2}} \int_{0}^{T}\left\{q \rho \bar{\rho} \lambda_{t} \xi_{t}+\frac{1}{2}\left(1-q \rho^{2}\right) \xi_{t}^{2}\right\} d t \\
& -\frac{1}{1-q \rho^{2}} \log H\left(0, Y_{0}\right) .
\end{aligned}
$$

Multiplying by $1-q \rho^{2}$ and using the stochastic representation (7) for $H\left(0, Y_{0}\right)$ gives, after some rearrangement,

$$
\begin{aligned}
& \frac{\exp \left(h K_{T}\right)}{E^{\widetilde{P}^{M}} \exp \left(h K_{T}\right)} \\
= & \mathcal{E}\left(-\frac{1-q \rho^{2}}{\sqrt{1-\rho^{2}}} \xi \cdot \widetilde{W}\right)_{T} \exp \left(-\frac{q \rho\left(1-q \rho^{2}\right)}{\sqrt{1-\rho^{2}}} \int_{0}^{T} \lambda_{t} \xi_{t} d t\right),
\end{aligned}
$$


where $h=-\frac{1}{2} q\left(1-q \rho^{2}\right)$. With $\chi$ defined as in the theorem, the right-hand side of this equation is precisely $d \widetilde{P}^{(q, \chi)} / d \widetilde{P}^{M}$, since we have

$$
\begin{aligned}
\frac{d \widetilde{P}^{(q, \chi)}}{d \widetilde{P}^{M}} & =\frac{d \widetilde{P}^{(q, \chi)}}{d P} / \frac{d \widetilde{P}^{M}}{d P} \\
& =\frac{\mathcal{E}[-q(\rho \lambda+\bar{\rho} \chi) \cdot \widetilde{W}]_{T}}{\mathcal{E}(-q \rho \lambda \cdot \widetilde{W})_{T}} \\
& =\mathcal{E}(-q \bar{\rho} \chi \cdot \widetilde{W})_{T} \exp \left(-q^{2} \rho \bar{\rho} \int_{0}^{T} \lambda_{t} \chi_{t} d t\right),
\end{aligned}
$$

which reproduces the right-hand side of (45) on using the definition of $\chi$ in the theorem.

For the case $q=1$ we have the striking corollary:

Corollary 3. The minimal entropy measure and minimal martingale measures, projected onto the sigma-field $\widetilde{\mathcal{F}}_{T}$, are related by the Esscher transform

$$
\frac{d \widetilde{Q}^{E}}{d \widetilde{Q}^{M}}=\frac{\exp \left(-\frac{1}{2}\left(1-\rho^{2}\right) K_{T}\right)}{E^{\widetilde{Q}^{M}} \exp \left(-\frac{1}{2}\left(1-\rho^{2}\right) K_{T}\right)} .
$$

See Monoyios (2005) for a proof of this result in a non-Markovian setting.

\section{Application to exponential hedging}

In this section we specialise to exponential utility, $U(x)=-\exp (-\alpha x)$, so $q=1$, and we modify the primal problem to allow for the sale of an $\widetilde{\mathcal{F}}_{T}$-measurable claim $B\left(Y_{T}\right) \equiv B$, representing the payoff of a European claim on $Y$. We assume the function $B(y)$ is such that all the expectations that appear below are well-defined. We apply a similar program to earlier sections, deriving a distortion representation for the primal problem, then exploring the ramifications for the dual problem. The proofs follow the same reasoning as for the problem with no random endowment, and so are omitted, leaving details as an exercise for the reader.

The objective in the primal problem is to maximise

$$
J_{B}(t, x, y ; \pi)=E\left[U\left(X_{T}-B\left(Y_{T}\right)\right) \mid X_{t}=x, Y_{t}=y\right] .
$$

The agent's primal value function is

$$
u_{B}(t, x, y):=\sup _{\pi \in \mathcal{A}} J_{B}(t, x, y ; \pi),
$$

with $u_{B}(T, x, y)=U(x-B(y))$. The class of admissible strategies $\mathcal{A}$ is defined in a similar manner to (17). 
By using the same methods as above, it is straightforward to establish the following distortion solution for $u_{B}$. The value function $u_{B}$ satisfies the same PDE as $u$, with modified terminal boundary condition.

Proposition 5. With exponential utility, $U(x)=-\exp (-\alpha x)$, the value function (46) is given by

$$
u_{B}(t, x, y)=U(x)\left(H_{B}(t, y)\right)^{1 /\left(1-\rho^{2}\right)},
$$

where $H_{B}:[0, T] \times \mathbb{R} \rightarrow \mathbb{R}^{+}$has the stochastic representation

$$
H_{B}(t, y)=E^{\widetilde{Q}^{M}}\left[e^{-\left(1-\rho^{2}\right)\left(\frac{1}{2} \int_{t}^{T} \lambda^{2}\left(Y_{s}\right) d s-\alpha B\left(Y_{T}\right)\right)} \mid Y_{t}=y\right] .
$$

The dual value function $v_{B}(t, \eta, y)$ is given by the Legendre transform

$$
v_{B}(t, \eta, y)=\sup _{x \in \mathbb{R}}\left(u_{B}(t, x, y)-x \eta\right) .
$$

The distortion representation for $u_{B}$ then implies that $v_{B}$ is given by

$$
v_{B}(t, \eta, y)=V(\eta)-\frac{\eta}{\alpha} \log \left(\left(H_{B}(t, y)\right)^{1 /\left(1-\rho^{2}\right)}\right),
$$

where $V(\eta)=(\eta / \alpha)(\log (\eta / \alpha)-1)$ is the convex conjugate of the exponential utility function. We recognise (49) as the analogue of (30) (for $q=1$ ) in the optimisation problem with random endowment.

The dual value function has the fundamental definition

$$
v_{B}(t, \eta, y):=\inf _{Q \in \mathcal{M}} E\left[V\left(\eta \frac{Z_{T}}{Z_{t}}\right)-\eta \frac{Z_{T}}{Z_{t}} B\left(Y_{T}\right) \mid Y_{t}=y\right] .
$$

For $t=0$, with $v_{B}(\eta) \equiv v_{B}(0, \eta, y)$, this is

$$
v_{B}(\eta)=V(\eta)+\frac{\eta}{\alpha} \inf _{Q \in \mathcal{M}}\left[d_{1}(Q, P)-\alpha E^{Q} B\left(Y_{T}\right)\right] .
$$

Then, equating (49) at $t=0$ with (50) gives the following result, the analogue of (28) for the problem with random endowment.

Proposition 6. The dual minimiser $Q^{B}$ achieving the infimum in (50) is related to $H_{B}\left(0, Y_{0}\right)$, and hence to the minimal measure $Q^{M}$, by

$$
\begin{aligned}
d_{1}\left(Q^{B}, P\right)-E^{Q^{B}}(\alpha B) & =-\frac{1}{1-\rho^{2}} \log E^{\widetilde{Q}^{M}} e^{-\left(1-\rho^{2}\right)\left(\frac{1}{2} K_{T}-\alpha B\right)} \\
& =-\frac{1}{1-\rho^{2}} \log H_{B}\left(0, Y_{0}\right) .
\end{aligned}
$$


Naturally, (51) reduces to (28) for $B=0$. The above proposition is new in that it links the measures $Q^{B}, Q^{M}$. Using it in the distortion solution for the primal value function allows us to reproduce more familiar duality results, as follows:

$$
\begin{aligned}
u_{B}(x) & =-\exp (-\alpha x)\left(H\left(0, Y_{0}\right)\right)^{1 /\left(1-\rho^{2}\right)} \\
& =-\exp \left(-\alpha x-\left[d_{1}\left(Q^{B}, P\right)-E^{Q^{B}}(\alpha B)\right]\right) \\
& =-\exp \left(-\alpha x-\inf _{Q \in \mathcal{M}}\left[d_{1}(Q, P)-\alpha E^{Q} B\right]\right),
\end{aligned}
$$

which is the fundamental duality in Delbaen et al. (2002).

\subsection{The dual control problem}

We may treat the dual problem with random endowment as a stochastic control problem over control processes $\psi \in \Psi$, in an analogous manner to the problem without random endowment.

Proposition 7. The dual minimiser $Q^{B}$ is given by

$$
\frac{d Q^{B}}{d P}=\mathcal{E}\left(-\lambda \cdot W-\xi^{B} \cdot W^{\perp}\right)_{T},
$$

with

$$
\xi_{t}^{B}=-\frac{b\left(Y_{t}\right)}{\sqrt{1-\rho^{2}}} \frac{\partial}{\partial y} \log H_{B}\left(t, Y_{t}\right), t \in[0, T],
$$

and $H_{B}(t, y)$ as in (48).

The next theorem is a novel representation for the solution of the dual stochastic control problem, the analogue of Theorem 2 for the problem with random endowment, and proven by similar methods. Define the functional

$$
C^{\psi, B}:=\frac{1}{2} \int_{0}^{T}\left(\lambda^{2}\left(Y_{t}\right)+\psi_{t}^{2}\right) d t-\alpha B
$$

Theorem 4. The dual problem with random endowment is the stochastic control problem to minimise

$$
d_{1}(Q, P)-\alpha E^{Q} B=E^{Q^{\psi}} C^{\psi, B}
$$

over processes $\psi \in \Psi$ satisfying $\int_{0}^{T} \psi_{t}^{2} d t<\infty$. The optimal control $\psi^{*}=$ : $\xi^{B}$ satisfies

$$
\exp \left(-\left(1-\rho^{2}\right) E^{Q^{\xi^{B}}} C^{\xi^{B}, B}\right)=E^{Q^{0}} \exp \left(-\left(1-\rho^{2}\right) C^{0, B}\right),
$$

where $Q^{0}=Q^{M}$ is the minimal martingale measure. 
We note that (53) is of the form

$$
\Phi^{(1)}\left(\inf _{\psi \in \Psi} E^{Q^{\psi}} C^{\psi, B}\right)=E^{Q^{0}} \Phi^{(1)}\left(C^{0, B}\right),
$$

where $\Phi^{(1)}(x)=\exp \left(-\left(1-\rho^{2}\right) x\right)$, as in Theorem 2 .

\subsection{A representation equation for the dual minimiser}

We derive a martingale representation identity for the dual minimiser $Q^{B}$, extending (34) for $q=1$ to the problem with random endowment, by considering the integral

$$
\int_{0}^{T} d\left(\log H_{B}\left(t, Y_{T}\right)\right)
$$

in a similar manner to the proof of Proposition 3.

Theorem 5. Define

$$
\begin{aligned}
L_{t}^{B} & :=\int_{0}^{t} \xi_{s}^{B} d W_{s}^{\perp}, \\
M_{t}^{B} & :=\int_{0}^{t} v_{s}^{B}\left(d W_{s}^{\perp}+\lambda\left(Y_{s}\right) d s\right),
\end{aligned}
$$

with

$$
\bar{\rho} v_{t}^{B}-\rho \xi_{t}^{B}=0,
$$

and where $\xi^{B}$ is given by (52). Then $M_{T}^{B}, L_{T}^{B}$ satisfy

$$
\frac{1}{2} K_{T}-\alpha B=M_{T}^{B}+L_{T}^{B}+\frac{1}{2}\left[L^{B}\right]_{T}+d_{1}\left(Q^{B}, P\right)-\alpha E^{Q^{B}} B .
$$

\subsection{Indifference pricing}

Define the indifference selling price (at time $t$, given $Y_{t}=y$ ) of the claim $B, P_{B}(t, y)$, by

$$
u_{B}\left(t, x+P_{B}(t, y), y\right)=u(t, x, y),
$$

where $u$ is the value function (18) when no claim is present. Note that we have anticipated the well-known property of indifference prices under exponential preferences, namely, that they do not depend on the initial cash endowment $x$. Using (23) and (47) in (54) gives the following representation for the indifference price. 


\section{Theorem 6.}

$$
\begin{aligned}
\exp \left(\alpha\left(1-\rho^{2}\right) P_{B}(t, y)\right) & =\frac{H_{B}(t, y)}{H(t, y)} \\
= & \frac{E^{\left.\widetilde{Q}^{M}\left[e^{-\left(1-\rho^{2}\right)\left(\frac{1}{2} \int_{t}^{T} \lambda^{2}\left(Y_{s}\right) d s-\alpha B\left(Y_{T}\right)\right.}\right) \mid Y_{t}=y\right]}}{E^{\widetilde{Q}^{M}}\left[e^{-\frac{1}{2}\left(1-\rho^{2}\right) \int_{t}^{T} \lambda^{2}\left(Y_{s}\right) d s} \mid Y_{t}=y\right]} .
\end{aligned}
$$

After the first version of this paper was written we discovered that, independently of this work, Grasselli and Hurd (2006) have produced this result. We can recast the above result into another form, in terms of the dual optimisers $Q^{B}, Q^{E}$ for the problems with and without random endowment, by setting $t=0$ and using (51) and (28) on the right-hand side, to give, for $P_{B} \equiv P_{B}\left(0, Y_{0}\right)$ :

$$
P_{B}=\sup _{Q \in \mathcal{M}}\left[E^{Q} B-\frac{1}{\alpha}\left(d_{1}(Q, P)-d_{1}\left(Q^{E}, P\right)\right)\right],
$$

which is the representation found in Delbaen et al. (2002), and from which well-known limits $\lim _{\alpha \rightarrow \infty} P_{B}=\sup _{Q \in \mathcal{M}} E^{Q} B$ and $\lim _{\alpha \rightarrow 0} P_{B}=E^{Q^{E}} B$ easily follow.

Finally, we note that for deterministic $K$ Theorem 6 reduces to

$$
\exp \left\{\left(1-\rho^{2}\right) \alpha P_{B}\right\}=E^{\widetilde{Q}^{M}} \exp \left\{\left(1-\rho^{2}\right) \alpha B\right\},
$$

which is the representation found in (Henderson (2002), Monoyios (2004), Musiela and Zariphopoulou (2004)).

\section{References}

Becherer, D. (2004): Utility-indifference hedging and valuation via reaction-diffusion systems. Proceedings of the Royal Society of London. Series A 460, 27-51

Bellini, F., Frittelli, M. (2002): On the existence of minimax martingale measures. Mathematical Finance 12, 1-21

Benth, F.E., Karlsen, K.H. (2005): A PDE representation of the density of the minimal entropy martingale measure in stochastic volatility markets. Stochastics 77, 109-137

Delbaen, F., Grandits, P., Rheinländer, T., Samperi, D., Schweizer, M., Stricker, C. (2002): Exponential hedging and entropic penalties. Mathematical Finance 12, 99-123

Föllmer, H., Schweizer, M. (1991): Hedging of contingent claims under incomplete information. In: Davis, M.H.A., Elliott, R.J. (eds.): Applied stochastic analysis. ( Stochastics Monographs 5) Gordon \& Breach, New York, pp. 389-414

Frittelli, M. (2000): The minimal entropy martingale measure and the valuation problem in incomplete markets. Mathematical Finance 10, 39-52

Goll, T., Rüschendorf, L. (2001): Minimax and minimal distance martingale measures and their relationship to portfolio optimization. Finance and Stochastics 5, 557-581 
Grasselli, M.R., Hurd, T.R. (2006): Indifference pricing and hedging for volatility derivatives. Preprint. Department of Mathematics and Statistics, McMaster University, Hamilton, Ontario

Henderson, V. (2002): Valuation of claims on nontraded assets using utility maximization. Mathematical Finance 12, 351-373

Henderson, V., Hobson, D.G., Howison, S., Kluge, T. (2005): A comparison of $q$-optimal option prices in a stochastic volatility model with correlation. Review of Derivatives Research 8, 5-25

Heston, S.L. (1993): A closed-form solution for options with stochastic volatility with applications to bond and currency options. The Review of Financial Studies 6, 327-343

Hobson, D.G. (2004): Stochastic volatility models, correlation, and the $q$-optimal measure. Mathematical Finance 14, 537-556

Kabanov, Y., Stricker, C. (2002): On the optimal portfolio for the exponential utility maximization: remarks to the six-author paper "Exponential hedging and entropic penalties". Mathematical Finance 12, 125-134

Karatzas, I., Lehoczky, J.P., Shreve, S.E., Xu, G.-L. (1991): Martingale and duality methods for utility maximization in an incomplete market. SIAM Journal on Control and Optimization 29, 702-730

Kramkov, D., Schachermayer, W. (1999): The asymptotic elasticity of utility functions and optimal investment in incomplete markets. The Annals of Applied Probability 9, 904 950

Monoyios, M. (2004): Performance of utility-based strategies for hedging basis risk. Quantitative Finance 4, 245-255

Monoyios, M. (2005): The minimal entropy measure and an Esscher transform in an incomplete market model. Preprint. Mathematical Institute, University of Oxford, Oxford

Musiela, M., Zariphopoulou, T. (2004): An example of indifference prices under exponential preferences. Finance and Stochastics 8, 229-239

Owen, M. (2002): Utility based optimal hedging in incomplete markets. The Annals of Applied Probability 12, 691-709

Pham, H. (2002): Smooth solutions to optimal investment models with stochastic volatilities and portfolio constraints. Applied Mathematics and Optimization 46, 55-78

Rheinländer, T. (2005): An entropy approach to the Stein and Stein model with correlation. Finance and Stochastics 9, 399-413

Schachermayer, W. (2001): Optimal investment in incomplete markets when wealth may become negative. The Annals of Applied Probability 11, 694-734

Schweizer, M. (1999): A minimality property of the minimal martingale measure. Statistics \& Probability Letters 42, 27-31

Stein, E.M., Stein, J.C. (1991): Stock price distributions with stochastic volatility: an analytic approach. The Review of Financial Studies 4, 727-752

Stoikov, S.F., Zariphopoulou, T. (2004): Optimal investments in the presence of unhedgeable risks and under CARA preferences. Preprint. Department of Mathematics, University of Texas, Austin

Tehranchi, M. (2004): Explicit solutions of some utility maximization problems in incomplete markets. Stochastic Processes and their Applications 114, 109-125

Zariphopoulou, T. (2001): A solution approach to valuation with unhedgeable risks. Finance and Stochastics 5, 61-82 